\title{
Cuidados de Enfermagem na prevenção do tromboembolismo venoso: revisão integrativa
}

\author{
Milara Barp ${ }^{1}$, \\ Viviane Santos Mendes Carneiro², \\ Kelle Vanessa Alvares Amaral', \\ Valéria Pagotto 4 , \\ Suelen Gomes Malaquias ${ }^{5}$
}

\section{RESUMO}

Este estudo buscou identificar na literatura científica quais os cuidados de enfermagem na prevenção do Tromboembolismo Venoso (TEV) em pacientes hospitalizados. Trata-se de uma revisão integrativa da literatura realizada nas bases de dados Public/Publish Medline (PUBMED) e Biblioteca Virtual em Saúde (BVS). Incluíram-se estudos em inglês, espanhol e português, publicados entre 2006-2016, que apresentassem ações de Enfermagem na prevenção do TEV nos pacientes hospitalizados, para a localização dos estudos utilizou-se o cruzamento dos termos "cuidados de enfermagem", "prevenção" e "tromboembolismo venoso". Predominaram os cuidados de enfermagem na prevenção do TEV categorizados em "implementação da avaliação do risco de TEV", "intervenções mecânicas e físicas" e "ensino ao paciente sobre TEV". Esses achados podem contribuir para sistematização da assistência de enfermagem pela ampliação e direcionamento das possibilidades de cuidados, além de favorecer autonomia do enfermeiro, subsidiar pesquisas de validação e reforçar a implementação de Enfermagem Vascular como área de especialidade.

Descritores: Cuidados de Enfermagem; Tromboembolia Venosa; Prevenção de Doenças; Enfermagem Baseada em Evidências.

\footnotetext{
${ }^{1}$ Enfermeira, Especialista em Urgência e Emergência pelo Programa de Residência Multiprofissional em Saúde do Hospital das Clínicas da Universidade Federal de Goiás. Goiânia, GO, Brasil. E-mail: milarabarp@hotmail.com.

${ }^{2}$ Enfermeira. Discente do Programa de Pós-Graduação em Atenção à Saúde, nível Mestrado, da Pontifícia Universidade Católica de Goiás. Goiânia, GO, Brasil. E-mail: vsm.mendes@gmail.com.

${ }^{3}$ Enfermeira. Discente do Programa de Pós-Graduação em Enfermagem, nível Mestrado, da Universidade Federal de Goiás. Goiânia, GO, Brasil. Email: kellealvares@gmail.com.

${ }^{4}$ Enfermeira, Doutora em Ciências da Saúde. Professora Adjunta da Faculdade de Enfermagem da Universidade Federal de Goiás. Goiânia, GO, Brasil. E-mail: valeriapagotto@gmail.com.

${ }^{5}$ Enfermeira, Doutora em Ciências da Saúde. Professora Adjunta da Faculdade de Enfermagem da Universidade Federal de Goiás. Goiânia, GO, Brasil. E-mail: sgmalaquias@gmail.com.
}

\section{Como citar esse artigo:}

Barp M, Carneiro VSM, Amaral KVA, Pagotto V, Malaquias SG. Cuidados de Enfermagem na prevenção do tromboembolismo venoso: revisão integrativa. Rev. Eletr. Enf. [Internet]. 2018 [acesso em: ];20:v20a14. Disponível em: https://doi.org/10.5216/ree.v20.48735. 


\section{INTRODUÇÃO}

Quando os trombos se formam no sistema venoso desencadeiam o tromboembolismo venoso (TEV), manifestado pela trombose venosa profunda (TVP) e tromboembolismo pulmonar (TEP) ${ }^{(1-2)}$, situações clínicas que podem ser rapidamente fatais ou propiciar incapacidades ou invalidez que provocam um alto impacto na saúde, econômico e social ${ }^{(3)}$.

Estima-se que uma em cada quatro mortes no mundo são decorrentes do TEV, sendo considerada a terceira causa de morte cardiovascular, atrás somente do infarto agudo do miocárdio e acidente vascular encefálico ${ }^{(1-2)}$.

No ambiente hospitalar, o tromboembolismo venoso é importante causa de morbidade e mortalidade, estima-se que $60 \%$ dos casos do TEV ocorram durante ou após a hospitalização, tornando-se uma das principais causas de óbito hospitalar evitável(1).

A tríade de Virchow que consiste em estase sanguínea, hipercoagulabilidade e dano vascular, explica a patogênese do TEV(1). Os fatores de risco para TEV podem ser hereditários/idiopáticos (trombofilias, história de TEV anterior) e adquiridos/provocados (idade avançada, comorbidades, imobilização, uso de cateteres venosos centrais, infecções, tratamento cirúrgico, quimioterapia, entre outros fatores já descritos na literatura científica) e individuos com vários fatores concomitantemente possuem maior risco de desenvolver o TEV ${ }^{(3-4)}$.

Considerando os diversos fatores de risco que pacientes hospitalizados possuem para o TEV, o enfermeiro é o profissional que está de modo contínuo e ininterrupto prestando assistência aos pacientes hospitalizados, portanto são fundamentais na identificação do risco de tromboembolismo venoso e na implementação das intervenções profiláticas ${ }^{(5-6)}$.

Nesse sentido, é necessário refletir sobre a definição de intervenção. Para a Classificação das Intervenções de Enfermagem ${ }^{(7)}$, Intervenção de Enfermagem é o tratamento elegido pelo enfermeiro a partir do conhecimento e raciocínio clínico, direcionado para melhorar os resultados do paciente

Porém, para a escolha de cuidados para prevenir o TEV se faz necessário uma assistência de Enfermagem baseada em evidências científicas, a fim de contribuir para ultrapassar uma prática ainda ineficiente no cuidado aos pacientes hospitalizados com risco de $\mathrm{TEV}^{(6)}$, como pode ser verificado em estudo multicêntrico que demonstrou a subutilização de programas de prevenção ao TEV hospitalar, com somente $60 \%$ dos participantes recebendo medidas profiláticas adequadas ${ }^{(8)}$, corroborando os dados que estimam que ainda existam 135 vezes mais casos de TEV em pacientes hospitalizados do que em outras populações fora do contexto hospitalar ${ }^{(1)}$.

Portanto o presente estudo tem como objetivo identificar ações de enfermagem para a prevenção do tromboembolismo venoso em pessoas em processo de hospitalização.

\section{METODOLOGIA}

Revisão integrativa da literatura realizada de acordo com etapas metodológicas propostas na literatura ${ }^{(9)} \mathrm{e}$ recomendações previstas no PRISMA Statement ${ }^{(10)}$.

Para definição da pergunta de pesquisa utilizou-se a estratégia $\mathrm{PICO}^{(11)}$ configurando-se à seguinte questão norteadora: "Quais as evidências disponíveis na literatura sobre o cuidado de enfermagem na prevenção do TEV em pacientes hospitalizados?" 
A busca dos artigos foi realizada nas seguintes bibliotecas: Biblioteca Virtual em Saúde e Public/PublishMedline (PUBMED). Utilizou-se o cruzamento dos termos "cuidados de enfermagem" AND "prevenção" AND "tromboembolismo venoso" para localização dos estudos.

Foram considerados critérios de inclusão estudos experimentais e descritivos nas línguas portuguesa, inglesa e espanhola, publicados entre 2006-2016. Excluíram-se cartas, editoriais, teses, dissertações, revisões, capítulos de livros e demais textos não científicos.

Os artigos foram avaliados inicialmente segundo o título e o resumo por dois pesquisadores independentes, que avaliaram a presença dos critérios de inclusão por meio do teste de relevância elaborado previamente. Nos casos de divergência sobre a inclusão ou exclusão dos artigos, foi feita leitura minuciosa e discussão do estudo na íntegra, sendo que não havendo consenso, uma terceira pesquisadora decidiu a inclusão ou exclusão dos estudos.

Em seguida realizou-se a extração dos dados dos artigos incluídos por meio de um formulário elaborado pelos autores, contendo os seguintes itens: identificação, objetivos, características metodológicas (tipo de estudo, características da amostra), itens referentes ao cuidado de enfermagem, desfecho, conclusões e limitações da pesquisa.

Para análise do nível de evidência dos estudos que compuseram a amostra final, foi utilizada a classificação por forças de evidência proposta pelo Joanna Briggs Institute ${ }^{(12)}$.

Após o levantamento dos cuidados de enfermagem para prevenção do TEV nos estudos incluídos, compararam-se à intervenção de enfermagem Precaução contra embolia, contida na Classificação de Intervenção de Enfermagem (NIC) $)^{(7)}$, para os diagnósticos da taxonomia NANDA-I ${ }^{(13)}$ Risco de Perfusão Tissular Periférica Ineficaz e Perfusão Tissular Periférica Ineficaz.

Esse processo foi realizado a partir de leitura minuciosa das intervenções da $\mathrm{NIC}^{(7)}$, para os diagnósticos mencionados e respectivas atividades, associando aos resultados dos estudos incluídos nesta revisão, por meio de análise simples de conteúdo, sem um delineamento metodológico específico, visto que não se configurava o objetivo deste estudo.

Ressalta-se, assim, que buscou-se essa comparação, a fim de organizar a apresentação dos resultados encontrados, a partir de um referencial de Enfermagem.

\section{RESULTADOS}

Inicialmente foram identificados 169 estudos, sendo 37 artigos excluídos por duplicidade e 55 estudos por não atenderem os critérios de inclusão. Após aplicação do teste de relevância sobre os títulos e resumos, 77 artigos foram selecionados para a leitura crítica na íntegra. Destes, 50 foram excluídos por não especificar o cuidado na prevenção do TEV como ação de Enfermagem e 19 por não se tratar de textos científicos, totalizando sete artigos (Figura 1).

Os estudos foram desenvolvidos nos Estados Unidos ( $n=2)$, Irã $(n=1)$, China $(n=1)$, Turquia $(n=1)$, Canadá $(n=1)$, Brasil $(n=1)$ e o principal idioma de divulgação foi a língua Inglesa $(n=7)$. Em relação aos autores, em todos $(100 \%)$ os estudos havia enfermeiros na autoria, em quatro (57\%) estudos havia a participação do profissional médico. Quanto ao perfil das amostras, quatro artigos se referiam aos pacientes hospitalizados em tratamento cirúrgico (57\%) e três artigos a pacientes clínicos e cirúrgicos (43\%). 
Figura 1: Fluxograma de seleção dos artigos.

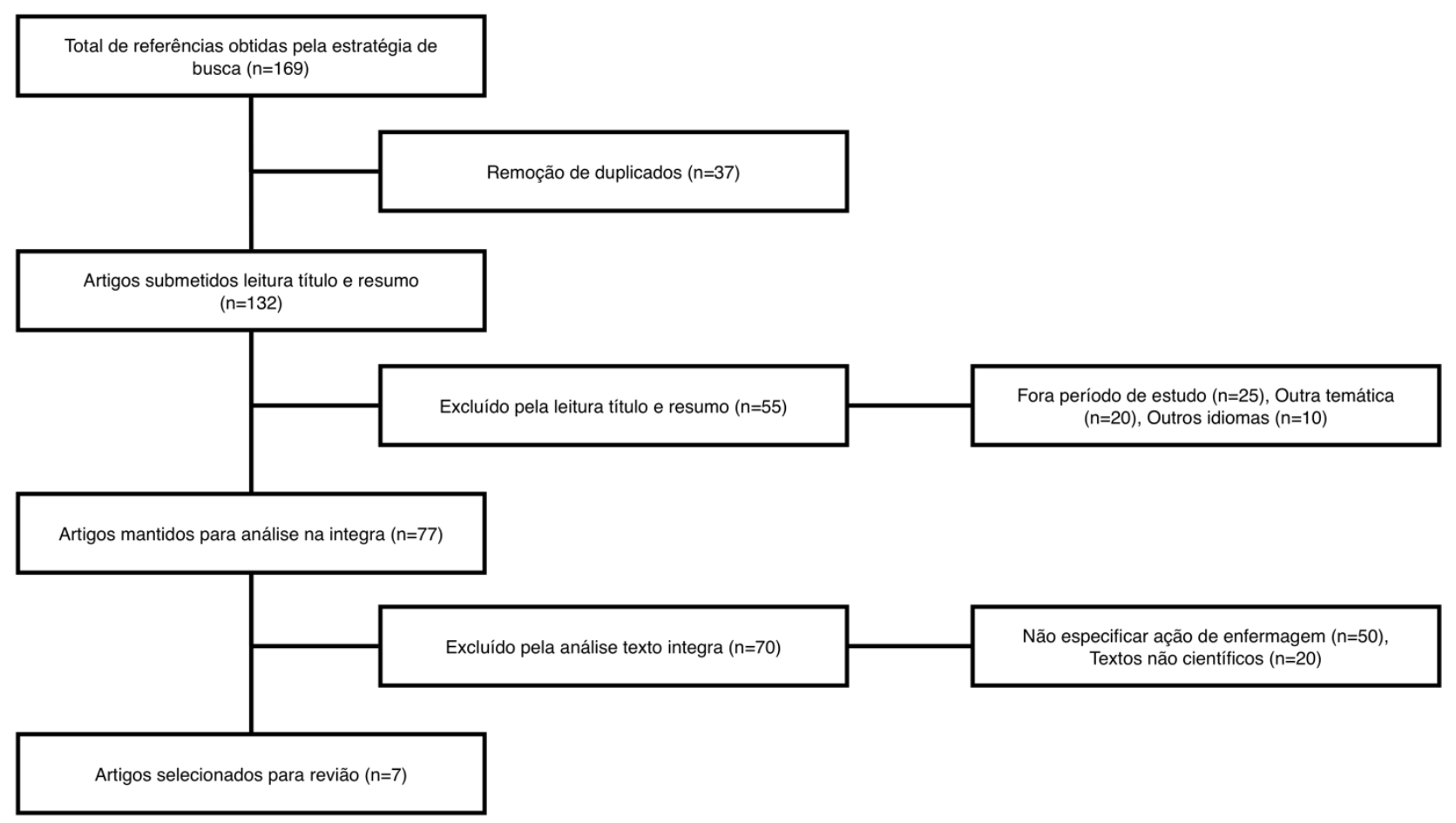

Quadro 1: Resultado da busca de artigos sobre cuidados de enfermagem na prevenção do tromboembolismo venoso, publicados entre 2006 e 2016, nas bibliotecas virtuais PubMed, BVS.

\begin{tabular}{|c|c|c|}
\hline Referência & Periódico & Desenho do estudo / Nível de evidência \\
\hline E1 - Ayhan et al., 2015 & Journal of Clinical Nursing & Ensaio Clínico randomizado / Nível de Evidência: 1 \\
\hline E2 - Hou et al., 2013 & Cancer Nursing & Ensaio Clínico randomizado / Nível de Evidência: 1 \\
\hline $\begin{array}{c}\text { E3 - Ayatollahzade-Isfahani et al., } \\
2013\end{array}$ & Journal Vascular Nursing & Ensaio Clínico randomizado / Nível de Evidência: 1 \\
\hline E4 - Le Sage, Mc Gee e Emed, 2008 & Journal Vascular Nursing & $\begin{array}{l}\text { Estudo quantitativo transversal / Nível de } \\
\text { Evidência: } 3\end{array}$ \\
\hline E5 - Castilho et al., 2010 & $\begin{array}{l}\text { Arquivos Brasileiros Ciências da } \\
\text { Saúde }\end{array}$ & $\begin{array}{c}\text { Estudo transversal observacional / Nível de } \\
\text { Evidência: } 3\end{array}$ \\
\hline E6 - Palamone et al., 2011 & Journal of Neuroscience Nursing & Estudo quase-experimental / Nível de Evidência: 2 \\
\hline E7 - Race e Collier, 2007 & Critical Care Nursing Quarterly & Série de casos / Nível de Evidência: 2 \\
\hline
\end{tabular}

Quanto aos objetivos dos estudos, observaram-se investigações concernentes à avaliação e estratificação do risco de TEV $(28,5 \%)$, terapia compressiva (14,3\%), eletroestimulação (14,3\%), posicionamento dos MMII (14,3\%), exercícios de amplitude de movimento $(14,3 \%)$ e conhecimento dos indivíduos sobre TEV e tromboprofilaxia (14,3\%) (Quadro 2).

Quanto às ações de Enfermagem, em uma das pesquisas os autores mencionam a aplicação de ferramenta para avaliar o risco de TEV como intervenção de Enfermagem, enfatizando o uso de uma escala de risco de TVP(14). Em outro estudo é citado a estratificação de risco de TEV proposta pela Sociedade Brasileira de Angiologia e Cirurgia vascular para nortear a escolha de medidas profiláticas ${ }^{(15)}$.

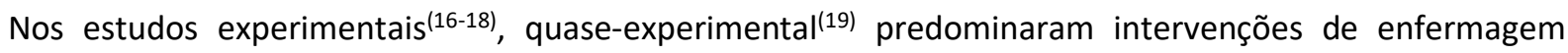
físicas e mecânicas para prevenção do TEV: terapia com meias elásticas compressivas, elevação dos MMII (membros inferiores), exercícios de amplitude de movimento para os MMII e eletroestimulação. Por fim, um dos estudos enfatiza o ensino ao paciente sobre TEV como medida a ser implementada pelo Enfermeiro ${ }^{(20)}$. 
Quadro 2: Síntese dos estudos incluídos de acordo com objetivo, método e principais resultados e conclusões.

\begin{tabular}{|c|c|c|c|}
\hline & Objetivo & Método & Principais resultados e conclusões \\
\hline \multirow{4}{*}{ E1 } & \multirow{4}{*}{$\begin{array}{l}\text { Comparar três tipos de meias elásticas de } \\
\text { diferentes níveis de compressão quanto a } \\
\text { eficácia de prevenir a TVP e o conforto que } \\
\text { oferecem aos pacientes em pós-operatório } \\
\text { cirurgias pélvicas e abdominais. }\end{array}$} & A amostra foi dividida em três grupos experimentais: & \multirow{4}{*}{$\begin{array}{l}\text { Não foi identificado TVP em nenhum dos pacientes dos três grupos } \\
\text { intervenção. Nos grupos III ( } 79,5 \%) \text { e grupo II ( } 52,1 \%) \text { os pacientes } \\
\text { apresentaram maiores desconfortos e dificuldade de aderir ao uso } \\
\text { das meias de compressão.Já no grupo I, as meias de compressão } \\
\text { foram relatadas como confortáveis pelos pacientes. Conclui-se que } \\
\text { meias elásticas de baixa compressão são tão eficazes como outros } \\
\text { tipos de meias elásticas que possuem outros graus de compressão e } \\
\text { altura. }\end{array}$} \\
\hline & & $\begin{array}{c}\text { Grupo I }(\mathrm{n}=73)=\text { Utilizaram meias elásticas de baixa compressão } \\
(15-18 \mathrm{mmHg}) \text { na altura do joelho. }\end{array}$ & \\
\hline & & $\begin{array}{l}\text { Grupo II }(n=73)=\text { Utilizaram meias elásticas baixa compressão (15- } \\
18 \mathrm{mmHg} \text { ) altura da coxa. }\end{array}$ & \\
\hline & & $\begin{array}{l}\text { Grupo III }(\mathrm{n}=73)=\text { Utilizaram meias elásticas de moderada } \\
\text { compressão }(20-30 \mathrm{mmHg}) \text { na altura do joelho. }\end{array}$ & \\
\hline \multirow{4}{*}{ E2 } & \multirow{4}{*}{$\begin{array}{l}\text { Testar a eficiência da estimulação elétrica em } \\
\text { pontos de acupuntura na prevenção pós- } \\
\text { cirúrgica da trombose venosa profunda em } \\
\text { pacientes idosos com tumor gastrointestinal } \\
\text { maligno. }\end{array}$} & Os participantes foram divididos em três grupos: & \multirow{4}{*}{$\begin{array}{l}\text { Verificou-se que os participantes que receberam eletroestimulação } \\
\text { elétrica (grupo T) apresentaram velocidade do fluxo sanguíneo } \\
\text { maior em comparação com os grupos C1 e C2. Quanto a viscosidade } \\
\text { sanguínea, o grupo T também apresentou valores menores } \\
\text { comparado ao grupo C1 e C2. Portanto, eletroestimulação mostra-se } \\
\text { um tratamento promissor na prevenção de trombose venosa } \\
\text { profunda em pacientes idosos com diagnóstico de tumor } \\
\text { gastrointestinal maligno. }\end{array}$} \\
\hline & & $\begin{array}{l}\text { Grupo Cl: receberam informações sobre elevação dos MMII, } \\
\text { realização de exercícios físicos na região inferior da perna durante } \\
\text { sete dias, três repetições ao dia. }\end{array}$ & \\
\hline & & $\begin{array}{l}\text { Grupo Cll: tratamento do grupo C1 associado ao uso de meias } \\
\text { elásticas compressivas no pós-operatório. }\end{array}$ & \\
\hline & & $\begin{array}{c}\text { Grupo T: mesmo tratamento do grupo C1 e eletroestimulação no } \\
\text { pós-operatório. }\end{array}$ & \\
\hline \multirow{3}{*}{ E3 } & \multirow{3}{*}{$\begin{array}{l}\text { Comparar o impacto que o decúbito dorsal } \\
\text { com elevação dos membros inferiores tem em } \\
\text { relação ao decúbito dorsal sem elevação na } \\
\text { prevenção da TVP em pacientes submetidos à } \\
\text { cirurgia de revascularização cardíaca. }\end{array}$} & $\begin{array}{l}\text { Grupo } 1 \text { ( } n=92): \text { Pacientes foram mantidos em decúbito dorsal sem } \\
\text { elevação de MMII }\end{array}$ & \multirow{3}{*}{$\begin{array}{l}\text { A TVP foi detectada em } 25 \text { (13,5\%) pacientes: } 17 \text { (18,4\%) decúbito } \\
\text { dorsal sem elevação e oito ( } 8,6 \%) \text { no grupo que recebeu a elevação } \\
\text { da perna ( } p=0,065) \text {. Não foi estatisticamente comprovado } \\
\text { associação entre o efeito do tipo de posição sobre a ocorrência de } \\
\text { TVP nos pacientes do estudo frente ao grande número de potenciais } \\
\text { fatores confusionais. No entanto, seu uso é um método seguro para } \\
\text { diminuir a estase venosa, sem provocar efeitos colaterias. }\end{array}$} \\
\hline & & $\begin{array}{c}\text { Grupo } 2 \text { ( } n=93 \text { ): Pacientes foram submetidos na posição decúbito } \\
\text { dorsal à elevação dos MMII ( } 30 \text { ㅇ) no pré-operatório, intraoperatório } \\
\text { e pós-operatório até a deambulação completa. }\end{array}$ & \\
\hline & & $\begin{array}{l}\text { Os dois grupos receberam antiplaquetário e anticoagulante e } \\
\text { receberam fisioterapia motora no pós-cirúrgico. }\end{array}$ & \\
\hline E4 & $\begin{array}{l}\text { Investigar o conhecimento dos pacientes } \\
\text { sobre tromboembolismo venoso e } \\
\text { tromboprofilaxia. }\end{array}$ & $\begin{array}{l}\text { Os pacientes foram avaliados por meio de questionário com } 16 \\
\text { questões objetivas e cinco questões subjetivas sobre: conhecimento } \\
\text { sobre tromboprofilaxia farmacológica, trombose venosa profunda e } \\
\text { embolismo pulmonar; de que forma receberam informações sobre } \\
\text { TEV; como desejariam ser informados sobre TEV no ambiente } \\
\text { hospitalar. }\end{array}$ & $\begin{array}{l}\text { Verificou-se que } 80 \% \text { da amostra estava ciente que recebia } \\
\text { medicação tromboprofilática, } 81.2 \% \text { dos pacientes responderam que } \\
\text { conheciam sobre a TVP e TEP. Quando interrogados sobre } \\
\text { sintomatologia, medidas profiláticas e complicações verificou-se } \\
\text { conhecimento deficiente. A relação entre TVP e TEP era } \\
\text { praticamente desconhecida. Um terço dos pesquisados referiram } \\
\text { que gostariam de receber mais informações pelo enfermeiro. }\end{array}$ \\
\hline
\end{tabular}




\begin{tabular}{|c|c|c|c|}
\hline & Objetivo & Método & Principais resultados e conclusões \\
\hline E5 & $\begin{array}{l}\text { Verificar por meio da avaliação de prontuários } \\
\text { de pacientes hospitalizados em unidade de } \\
\text { terapia intensiva os fatores de risco de TVP, } \\
\text { avaliar as medidas profiláticas adotadas aos } \\
\text { pacientes em risco mediante prescrições } \\
\text { médicas e de enfermagem. }\end{array}$ & $\begin{array}{c}\text { Realizou-se a leitura dos prontuários em uma única vez. Conforme } \\
\text { se apresentavam no momento da coleta. Identificaram-se os fatores } \\
\text { de risco para TEV e analisou-se conforme o algoritmo de } \\
\text { classificação do risco de TEV recomendada pela SBACV Sociedade } \\
\text { Brasileira de Angiologia e Cirurgia Vascular (2005). Levantaram-se } \\
\text { ainda as condutas médicas e de enfermagem que estavam sendo } \\
\text { realizadas para prevenir o TEV. }\end{array}$ & $\begin{array}{l}\text { Dos pacientes cirúrgicos } 76 \% \text { foram estratificados como de alto risco } \\
\text { e, todos pacientes clínicos em UTI foram considerados de alto risco. } \\
\text { Quanto ao tratamento medicamentoso, } 78 \% \text { dos pacientes clínicos e } \\
64,8 \% \text { dos pacientes cirúrgicos recebiam heparina não fracionada } \\
\text { (HNF). Somente um paciente realizou uso de botas pneumáticas } \\
\text { para prevenir TEV. Foi encontrado na prescrição de enfermagem } \\
\text { somente o item avaliar a perfusão periférica. A movimentação no } \\
\text { leito era realizada somente nos períodos de mudança de decúbito. }\end{array}$ \\
\hline E6 & $\begin{array}{l}\text { Verificar a efetividade de exercícios de } \\
\text { amplitude de movimento dos pés e tornozelos } \\
\text { na prevenção de trombose venosa profunda } \\
\text { em pacientes internados na unidade de } \\
\text { terapia intensiva neurológica. }\end{array}$ & $\begin{array}{l}\text { Os pacientes admitidos na UTI neurocirúrgica receberam medidas } \\
\text { profiláticas (medicações tromboprofiláticas, meias de compressão } \\
\text { durante o período de imobilidade, deambulação precoce etc.) e, } \\
\text { eram ensinados e estimulados a realizar exercícios de dorsiflexão e } \\
\text { flexão plantar, seguido de rotação medial e lateral do pé, } 10 \text { vezes a } \\
\text { cada hora. }\end{array}$ & $\begin{array}{l}\text { Não houve diferença nas taxas de TVP nos pacientes que receberam } \\
\text { os exercícios de amplitude durante o tempo de estudo comparado } \\
\text { aos que somente receberam o tratamento padrão. Nos pacientes } \\
\text { que concordaram em aderir ao programa de exercícios e realizavam } \\
\text { o exercício de amplitude pé/tornozelo a ocorrência de TVP foi } \\
\text { menor ( } 38,7 \%) \text { quando comparado aos pacientes que não } \\
\text { executavam adequadamente }(58,4 \%) \text {. }\end{array}$ \\
\hline E7 & $\begin{array}{l}\text { Revisar os casos clínicos de pacientes com } \\
\text { fatores de risco para TVP hospitalizados, } \\
\text { discutir sobre a estratificação do risco e } \\
\text { medidas profiláticas adotadas. }\end{array}$ & $\begin{array}{l}\text { Realizou-se a análise dos casos levantando fatores positivos para } \\
\text { TVP, conforme a escala de estratificação de risco de Caprini, } \\
\text { verificou-se ainda o manejo clínico adotado aos pacientes para } \\
\text { prevenção TEV. }\end{array}$ & $\begin{array}{l}\text { No caso 1, a ausência de ferramenta para reconhecer o risco fez com } \\
\text { que fatores positivos para TVP passassem despercebidos. O paciente } \\
\text { não recebeu tromboprofilaxia durante internação, com isso, recebeu } \\
\text { alta médica e rapidamente foi readmitido com quadro de TEP, } \\
\text { evoluindo ao óbito. No caso } 2 \text {, o paciente possui altíssimo risco para } \\
\text { TVP, recebeu tromboprofilaxia mecânica por meio de dispositivo de } \\
\text { compressão intermitente, não desenvolveu TVP. A aplicação da } \\
\text { escala proposta por Caprini permite rastrear pacientes em risco e } \\
\text { auxiliar na escolha da medida profilática mais adequada para evitar } \\
\text { complicações tromboembólicas. }\end{array}$ \\
\hline
\end{tabular}


Observam-se contribuições importantes das intervenções apresentadas nos estudos para prevenção do TEV, entre as quais destacam-se: repercussões positivas da eletroestimulação, uso de meias elásticas compressivas, elevação dos MMII, exercícios de amplitude de movimento e de ações educativas acerca do TEV. Também observaram-se fragilidades em relação ao uso de ferramentas de estratificação do risco de TVP.

Para ilustrar a associação entre as recomendações descritas na literatura e as indicadas pela área de Enfermagem, utilizou-se a Classificação de Intervenções de Enfermagem $(\mathrm{NIC})^{(7)}$, frequentemente, utilizada para nortear as prescrições dos enfermeiros.

Dessa forma, observou-se que a intervenção "Precauções contra embolia" não está incluída como prioritária para os diagnósticos “Risco de perfusão tissular periférica ineficaz" e "Perfusão tissular periférica ineficaz"(13), os quais são os mais correspondentes à situação clínica de TEV.

Assim, observou-se a limitada associação entre os resultados identificados nos estudos e as atividades descritas para intervenções no contexto de TEV a pacientes hospitalizados (Quadro 3).

Quadro 3: Comparação entre os cuidados identificados nos estudos analisados com os contidos na intervenção “Precauções contra embolia" da Classificação de Intervenções de Enfermagem (NIC)(7).

\begin{tabular}{|c|c|}
\hline $\begin{array}{l}\text { Cuidados de enfermagem para prevenção do TEV, segundo } \\
\text { os estudos incluídos na revisão: }\end{array}$ & $\begin{array}{l}\text { Cuidados correspondentes da intervenção “Precauções contra } \\
\qquad{\text { embolia" }(\mathrm{NIC})^{(7)} \text { : }}\end{array}$ \\
\hline \multirow{2}{*}{$\begin{array}{c}\text { Realizar a avaliação e estratificação do risco de TEV em } \\
\text { pacientes hospitalizados(14-15) }\end{array}$} & $\begin{array}{l}\text { Obter histórico detalhado da saúde do paciente a fim de } \\
\text { determinar seus fatores de risco }\end{array}$ \\
\hline & $\begin{array}{c}\text { Avaliar a presença da tríade de Virchow: estase venosa, } \\
\text { hipercoagubilidade, traumatismo resultante de dano intimal }\end{array}$ \\
\hline $\begin{array}{l}\text { Indicar, implementar o uso de meias compressivas, } \\
\text { conforme prévia estratificação do risco de TEV(16) }\end{array}$ & $\begin{array}{l}\text { Aplicar meia elástica de compressão gradual ou mangas de } \\
\text { compressão para reduzir o risco de TVP ou evitar sua } \\
\text { recorrência, conforme política e protocolo organizacional }\end{array}$ \\
\hline $\begin{array}{c}\text { Avaliar diariamente a coloração da pele, perfusão periférica } \\
\text { dos membros recebendo tratamento compressivo(16) }\end{array}$ & \multirow{2}{*}{$\begin{array}{l}\text { Remover meia elástica de compressão gradual e dispositivo de } \\
\text { compressão pneumática intermitente por } 15 \text { a } 20 \text { minutos a } \\
\text { cada oito horas ou conforme política e protocolo organizacional }\end{array}$} \\
\hline $\begin{array}{c}\text { Avaliar o nível de conforto com o uso e, garantir a } \\
\text { compressão adequada(16) }\end{array}$ & \\
\hline Aplicação da eletroestimulação em pontos de acupuntura(17) & Não há cuidado correspondente \\
\hline $\begin{array}{l}\text { Posicionar o paciente decúbito dorsal com elevação MMII a } \\
30 \text { ㅇ durante intraoperatório e pós-operatório(18) }\end{array}$ & $\begin{array}{c}\text { Elevar qualquer membro que se suponha estar afetado no } \\
\text { mínimo } 20 \text { - acima do nível do coração para aumentar o retorno } \\
\text { venoso }\end{array}$ \\
\hline $\begin{array}{l}\text { Ensinar e supervisionar o paciente na realização de } \\
\text { exercícios de dorsiflexão e flexão plantar, seguido de } \\
\text { rotação medial e lateral do pé, } 10 \text { repetições a cada hora, } \\
\text { até a total deambulação do paciente pós-cirúrgico(19) }\end{array}$ & $\begin{array}{l}\text { Encorajar o paciente a flexionar e estender o pé e pernas pelo } \\
\qquad \text { menos } 10 \text { vezes a cada hora }\end{array}$ \\
\hline $\begin{array}{l}\text { Ensino aos pacientes hospitalizados sobre TEV, medidas } \\
\text { preventivas e complicações da doença(20) }\end{array}$ & $\begin{array}{l}\text { Orientar o paciente e familiares quanto às precauções } \\
\text { necessárias }\end{array}$ \\
\hline \multirow{4}{*}{ Ensino ao paciente em uso de tromboprofiláticos(20) } & $\begin{array}{c}\text { Orientar o paciente e seus familiares sobre todos os } \\
\text { anticoagulantes de baixa dosagem profiláticos e/ou } \\
\text { medicamento antiplaquetário }\end{array}$ \\
\hline & $\begin{array}{l}\text { Orientar paciente a relatar sangramentos excessivos, feridas } \\
\text { anormais, dores ou edema incomuns, cianose e dor nos } \\
\text { pés/pododáctilos, úlceras ou manchas brancas na cavidade oral }\end{array}$ \\
\hline & $\begin{array}{l}\text { Orientar o paciente a usar o anticoagulante no mesmo horário } \\
\text { todos os dias e para não dobrar a dose no dia seguinte caso ele } \\
\text { esqueça da medicação }\end{array}$ \\
\hline & $\begin{array}{l}\text { Orientar o paciente a consultar um profissional de saúde antes } \\
\text { de usar qualquer medicamento ou preparo à base de plantas, } \\
\text { antes de escolher marcadas de medicamentos e antes de } \\
\text { interromper uso desses }\end{array}$ \\
\hline
\end{tabular}




\section{DISCUSSÃO}

Mediante o achado de reduzido número de publicações direcionadas para cuidados de enfermagem na prevenção do tromboembolismo venoso (TEV), assim como o maior enfoque aos cuidados para o tratamento de feridas de etiologia vascular provenientes de desordem tromboembólica, é preciso considerar a necessidade de direcionar a prática também para intervenções que promovam saúde e previnam complicações, entre as quais as úlceras vasculares são prevalentes e demandam cuidados semelhantes para prevenção de recorrência(21).

No entanto, úlceras vasculares, apesar da morbidade expressiva, não representam a única complicação do TEV, sendo que no contexto da hospitalização, embolia pulmonar e outros eventos isquêmicos periféricos diversos, são extremamente preocupantes pelo risco de morte ${ }^{(1-4)}$.

Apesar de existirem iniciativas, principalmente em países da América do Norte, Europa e Ásia, voltadas para prevenção do TEV, que visam reduzir mortes e incapacidades provocadas pela doença ${ }^{(2)}$, observa-se reduzido número de periódicos e associações de enfermagem voltados para a área vascular, o que demonstra a necessidade de fortalecer a formação de enfermagem específica nessa área, que, consequentemente, contribuirá com abordagem direcionada e assertiva para prevenção de TEV.

Outro aspecto levantado é a ausência de estudos com amostras de pacientes exclusivamente clínicos. Sabese que $50 \%-70 \%$ dos casos de TVP e TEP fatal ocorrem em pacientes hospitalizados em situações de doenças clínicas $^{(14)}$

Como em outros estudos que buscaram associação de recomendações da literatura especializada e intervenções da Classificação de Intervenção de Enfermagem (NIC) ${ }^{(21)}$, observou-se, apesar da pertinência dos cuidados apresentados na NIC ${ }^{(7)}$, limitação dessa taxonomia no que se refere ao atendimento específico, como no caso dos pacientes com risco de desenvolver TEV, fato este que indica a necessidade de busca permanente à literatura especializada para maior abrangência das intervenções.

Observa-se, ainda, que no diagnóstico "Risco de perfusão tissular periférica ineficaz", definido como "vulnerabilidade a uma redução da circulação sanguínea periférica, que pode comprometer a saúde", não havia fator de risco que atribuísse à situação de hospitalização. Esse achado reforça a sugestão de que a atuação do enfermeiro no contexto de atendimento a pacientes hospitalizados em risco de TEV, ainda encontra-se pouco estabelecida entre as taxonomias investigadas, bem como refletem a prática clínica no que se refere à sistematização da assistência de enfermagem em nível institucional.

Nesse sentido, a $\mathrm{NIC}^{(7)}$ propõe, a partir de julgamento de profissionais com expertise na área, que para a intervenção "Precauções contra embolia" a formação mínima necessária para execução seja de profissional enfermeiro, e com tempo médio de 16 a 30 minutos para implementação de todas as atividades descritas.

Ao considerar a inclusão dos cuidados identificados nos estudos dessa revisão, é possível extrapolarem-se o tempo para execução, assim como a formação mínima, visto serem cuidados específicos, como eletroestimulação ${ }^{(17)}$ e ensino/supervisão de programa de exercícios com membros inferiores ${ }^{(19)}$.

Por outro lado, ressalta-se a relevância das informações apresentadas pela NIC para estimular o aprimoramento da sistematização da assistência nessa área de atuação de enfermagem.

Apesar disso, há de se considerar o número restrito de pesquisas experimentais identificadas neste estudo, a verificação da eficácia das intervenções é comprometida. Além disso, houve grande heterogeneidade das 
populações estudadas e, escassez de informações quanto às características das amostras, como informações sobre fatores de risco para TEV. Observou-se uma descrição pouco detalhada das medidas profiláticas adotadas aos grupos estudados, dificultando a análise criteriosa das intervenções.

Desta forma, para otimizar a análise dos dados referentes às intervenções identificadas nos estudos incluídos nesta revisão, propôs-se a apresentação em categorias elaboradas conforme às formas de atuação da enfermagem na prevenção do TEV.

\section{Categoria 1: Implementação da estratificação do risco para TEV: implicações ao enfermeiro}

A realização da avaliação do grau de risco, mencionada nos estudos brasileiro e americano ${ }^{(14-15)}$ contribui para orientar a elaboração e implementação de intervenções individualizadas que previnem o desenvolvimento do TEV, reduzindo o tempo de internação, número de readmissões e gastos hospitalares devido às complicações decorrentes dos eventos tromboembólicos ${ }^{(5,14-15)}$.

Porém, a rotina da avaliação do risco de TEV ainda é pouco realizada pelo enfermeiro ${ }^{(14-15)}$. Entre as barreiras que dificultam a prática está a falta de conhecimento do Enfermeiro, a ausência de protocolos institucionais e de ferramentas que auxiliem a avaliação do risco de TEV.

A avaliação do risco de TEV deve ser realizada na admissão e ao longo da internação, preferencialmente a cada 72 horas, considerando as várias fases e mudanças que ocorrem ao longo do processo de internação, que aumentam ou diminuem os fatores para o TEV(6,14).

Para auxiliar na avaliação podem ser utilizados instrumentos, tais como, o escore de risco de Caprini, ferramenta que avalia fatores de risco para TEV e classifica o paciente em muito baixo, baixo, moderado, alto ou altíssimo risco de desenvolver a TVP. De acordo com o resultado da estratificação há recomendações de medidas profiláticas que devem ser implementadas pela equipe de saúde ${ }^{(15,22-23)}$.

No Brasil, entidades médicas publicaram diretrizes para prevenção do TEV, contendo o algoritmo para avaliação da necessidade de profilaxia em pacientes clínicos e cirúrgicos, em que traz recomendações de medidas profiláticas conforme classificação do risco. Porém, observa-se subutilização pela equipe multidisciplinar dessa diretriz, bem como condutas de prevenção do TEV que não estão sendo realizadas conforme preconizadas ${ }^{(15,24)}$.

A subutilização de diretrizes é descrita também em estudo ${ }^{(25)}$ com 236 pacientes internados, destes $80 \%$ apresentavam alto risco de TEV, e 34,9\% recebiam condutas profiláticas apropriadas. Com a implementação de protocolo de prevenção do TEV, $43,2 \%$ dos pacientes passaram a receber profilaxia adequada. Em outro estudo ${ }^{\text {(26- }}$ 27), com a implementação da estratificação de risco de Caprini para nortear as medidas profiláticas a incidência de TVP em pacientes cirúrgicos diminuiu $84 \%$ e de TEP reduziu $55 \%$.

\section{Categoria 2: Intervenções mecânicas e físicas para prevenção do TEV}

A aplicação de meias elásticas compressivas graduadas foi a intervenção que demonstrou prevenir o TEV em todos pacientes cirúrgicos quando associado a outras modalidades preventivas ${ }^{(16)}$. Resultado semelhante foi encontrado em estudo que verificou que somente $4 \%$ dos pacientes desenvolveram TVP quando o uso de meias compressivas estava associada a outro método profilático, tais como anticoagulantes, mudança de decúbito e 
exercícios de amplitude de movimento, enquanto $16 \%$ dos pacientes adquiriram TVP com o uso isolado do dispositivo(16,28).

As meias elásticas atuam impulsionando os músculos da panturrilha, diminuindo o diâmetro das veias centrais levando ao aumento da velocidade e volume do fluxo sanguíneo que retorna ao coração(28-30). São indicadas para tratamento profilático para TEV em pacientes hospitalizados, classificados como baixo risco de desenvolver TEV, moderado risco nos casos em que a terapia anticoagulante está contraindicada pelo risco de evento hemorrágico ${ }^{(24)}$ e, em pacientes com alto risco de TEV associadas ao uso de medidas farmacológicas ${ }^{(23)}$.

O enfermeiro implementa, orienta e acompanha o paciente em tratamento com meias compressivas ${ }^{(5-6,16)}$. Na escolha do dispositivo o gradiente de pressão deve ser mais elevado na região do tornozelo, panturrilhas e menor na região da coxa, geralmente com compressão mínima de 20 mmHg para o efeito de evitar o TEV(16) Meias na altura do quadril costumam provocar maior desconforto e são de difícil manuseio para realizar os cuidados diários, na altura do joelho e da coxa costumam ser mais bem toleradas e, eficazes na prevenção da TVP(16,31).

Embora o enfermeiro possa recomendar o uso de meias elásticas, há de se ressaltar a necessidade de que este profissional seja devidamente capacitado para indicar e acompanhar os indivíduos nessa terapêutica, vistos os riscos possíveis da implementação desta conduta.

O enfermeiro deve realizar avaliação diária do paciente em uso de método compressivo, principalmente em regiões de proeminências ósseas e em pacientes com diminuição da sensibilidade nos membros, verifica-se a presença de palidez na pele, perda da integridade, marcações pelo excesso de compressão, relato de dor ou desconforto ${ }^{(16,31)}$.

Em relação às intervenções físicas de fácil aplicação e custo baixo estão a utilização de exercícios ativos e passivos para os membros inferiores, especialmente aqueles que envolvem os músculos da panturrilha, que diminuem o refluxo venoso, reduzindo a formação de $\operatorname{trombos}^{(19,31)}$.

De modo semelhante, o posicionamento do paciente em decúbito dorsal com membros inferiores elevados em ângulo de 30 o ou níveis acima do coração proporcionam repercussões como a diminuição do edema, da estase venosa e, consequente menor incidência de TVP. A elevação dos MMII deve ser realizada até o retorno completo da deambulação do indivíduo(18,31).

Em um dos estudos mencionou-se sobre os benefícios da utilização de terapia complementar associada ao tratamento convencional, o estudo aplica eletroestimulação em pontos de acupuntura nos MMII, desencadeando a redução da viscosidade sanguínea e aumento da velocidade do fluxo sanguíneo na região que recebeu o estímulo elétrico de baixa voltagem ${ }^{(17)}$.

O uso da eletroestimulação transcutânea desencadeia impulsos elétricos com frequência capaz de gerar movimento involuntário de grupos musculares, essas contrações quando em membros inferiores desencadeiam melhora no retorno venoso. O uso da estimulação elétrica costuma ser opção terapêutica em pacientes que não toleram o uso de terapia compressiva e nos pacientes com contraindicações de anticoagulantes, pelo risco de $\operatorname{hemorragia}^{(17,32)}$.

Apesar das comprovações dos benefícios das medidas físicas e mecânicas verifica-se barreiras que parecem impedir a implementação desses cuidados pela Enfermagem, tais como a falta de protocolos padronizados direcionados à clientela atendida, bem como a falta de treinamento para o uso das ferramentas, a ausência de 
dispositivos compressivos disponíveis para aplicar aos pacientes e falta de tempo são também descritos como empecilhos para realizar ações na prevenção do $\operatorname{TEV}^{(7)}$.

\section{Categoria 3: Ensino ao paciente sobre TEV}

Estudo realizado no Canadá(20) identificou que os pacientes hospitalizados desconhecem principalmente informações referentes aos sinais e sintomas do TEV, gravidade das complicações tromboembólicas e as formas de prevenção. Com os baixos níveis de conhecimento sobre TVP e TEP há uma baixa adesão de medidas profiláticas $^{(20,31)}$.

A educação em saúde compõe uma das atribuições do enfermeiro, porém em função das demandas técnicas e administrativas essa ação fica limitada. A prática educativa ocorre de forma verticalizada, restrita à orientações específicas principalmente durante a admissão e alta com fragilidades, na validação do processo de ensino-aprendizagem com os pacientes ${ }^{(20,33)}$.

O enfermeiro que consegue desempenhar sua função de ensino ao paciente com risco de TEV de forma mais ativa parece obter resultados satisfatórios, como foi verificado quando utilizou-se materiais explicativos ${ }^{(20)} \mathrm{e}$ vídeos $^{(34)}$ para conscientizar pacientes sobre o risco de TEV. O uso do recurso de vídeo sobre o TEV fez com que $83 \%$ das perguntas sobre fatores de risco, sintomatologia e medidas profiláticas fossem respondidas corretamente pelos pacientes hospitalizados, enquanto somente $62 \%$ das perguntas foram corretamente respondidas pelos pacientes que não receberam a estratégia de vídeo ${ }^{(34)}$.

O esclarecimento da doença e dos cuidados preventivos, tais como os benefícios da deambulação precoce e da terapia compressiva, podem estimular os pacientes a iniciar precocemente às intervenções ${ }^{(14)}$, resultados semelhantes podem ser verificados com os pacientes hipertensos, que, quando recebem estratégias de informação sobre a doença e tratamento, apresentam maior adesão ao tratamento(35).

O papel do enfermeiro perpassa o assistir e gerenciar, sendo o processo educativo uma atribuição inerente ao ser-fazer do enfermeiro. Pacientes, familiares e a própria equipe de enfermagem e agentes de saúde, estão sob sua responsabilidade ${ }^{(33)}$. Ressalta-se a importância da equipe de enfermagem ser permanentemente capacitada e supervisionada pelo enfermeiro para realizar cuidados de prevenção ao TEV.

Portanto, educar compreende-se como elemento do cuidar, por potencializá-lo e suscitar intervenções de forma construtivo-reflexiva, singular-plural, dinâmico-flexível. Dessa forma, contribui-se com a qualidade da assistência aos pacientes hospitalizados, promovendo valorização e ampliação da autonomia da enfermagem ${ }^{(33)}$.

\section{CONSIDERAÇÕES FINAIS}

Entre as medidas preventivas identificadas neste estudo, nas quais a Enfermagem pode intervir, destacamse: exercicíos de amplitude de movimento, terapia compressiva, mobilização precoce, posicionamento de MMII, eletroestimulação e ensino do indivíduo quanto ao risco de TEV, suas repercussões e estratégias para prevenção.

Embora os resultados apontem diferentes intervenções de Enfermagem para prevenir o TEV nos pacientes hospitalizados, a documentação de experiências de enfermeiros na prevenção do TEV ainda se mostra escassa, especialmente no Brasil. Esse fato aponta para reflexões sobre possíveis contribuições da organização e 
reconhecimento, no cenário nacional, da Enfermagem Vascular como área de especialidade, distinta das áreas relacionadas à dermatologia e/ou tratamento de feridas, assim como em outros países.

Dessa forma, é possível estabelecer investimentos mais direcionados e sistematizados nessa área, o que favorece o fortalecimento e autonomia do enfermeiro e consequentemente, melhorias para a assistência a essa clientela.

A implementação dos cuidados para prevenção do TEV identificados neste estudo, oferece subsídios para a realização do processo de enfermagem a pacientes hospitalizados com risco de perfusão tissular periférica ineficaz, contribuindo com a sistematização da asssistência, assim como à produção do conhecimento nesta área.

Ressalta-se a importância de pesquisas futuras de enfermagem que validem esses cuidados nas diversas populações, com seus perfis distintos, no contexto da hospitalização, como também extrapolando essas instituições, visto que há indivíduos com risco semelhante para TEV na Atenção Domiciliar, instituições de longa permanência, entre outros.

\section{REFERÊNCIAS}

1. Jha AK, Larizgoitia I, Audera-Lopez C, Prasopa-Plaizier N, Waters H, Bates DW. The global burden of unsafe medical care: analytic modeling of observational studies. BMJ Qual Saf [Internet]. 2013 [acesso em: 16 ago. 2018];22(10):809-15. Disponível em: https://doi.org/10.1136/bmjqs-2012-001748.

2. ISTH Steering Committee for World Thrombosis Day. Thrombosis: a major contributor to the global disease burden. J Thromb Haemost [Internet]. 2014 [acesso em: 16 ago. 2018];12(10):1580-90. Disponível em: https://doi.org/10.1111/jth.12698.

3. Wendelboe AM, McCumber M, Hylek EM, Buller H, Weitz J, Raskob G. Global public awareness of venous thromboembolism. J Thromb Haemost [Internet]. 2015 [acesso em: 16 ago. 2018];13(8):1365-71. Disponível em: https://doi.org/10.1111/ith.13031. 4. Tsai J, Grant AM, Beckman MG, Grosse SD, Yusuf HR, Richardson LC. Determinants of venous thromboembolism among hospitalizations of US adults: a multilevel analysis. PLoS One [Internet]. 2015 [acesso em: 16 ago. 2018];10(4):e0123842. Disponível em: https://doi.org/10.1371/journal.pone.0123842.

5. Gaston W, White S. Venous thromboembolism (VTE) risk assessment: Rural nurses' knowledge and use in a rural acute care hospital. Int J Nurs Pract [Internet]. 2013 [acesso em: 16 ago. 2018];19(1):60-4. Disponível em: https://doi.org/10.1111/ijn.12028. 6. Lee JA, Grochow D, Drake D, Johnson L, Reed P, van Servellen G. Evaluation of hospital nurses' perceived knowledge and practices of venous thromboembolism assessment and prevention. J Vasc Nurs [Internet]. 2014 [acesso em: 16 ago. 2018];32(1):1824. Disponível em: https://doi.org/10.1016/j.jvn.2013.06.001.

7. McCloskey JC, Bulecheck GM. Classificação das Intervenções de Enfermagem. 3ạ ed. Porto Alegre: Artmed; 2004.

8. Spyropoulos AC, Anderson FA Jr, FitzGerald G, Decousus H, Pini M, Chong BH, et al. Predictive and associative models to identify hospitalized medical patients at risk for VTE. Chest [Internet]. 2011 [acesso em: 16 ago. 2018];140(3):706-14. Disponível em: https://doi.org/10.1378/chest.10-1944.

9. Mendes KDS, Silveira RCCP, Galvão CM. Revisão integrativa: método de pesquisa para a incorporação de evidências na saúde e na enfermagem. Texto contexto - enferm [Internet]. 2008 [acesso em: 16 ago. 2018];17(4):758-64. Disponível em:

https://doi.org/10.1590/S0104-07072008000400018.

10. Moher D, Liberati A, Tetzlaff J. Preferred reporting items for systematic reviews and meta-analyses: the PRISMA statement. PLoS Med [Internet]. 2009 [acesso em: 16 ago. 2018];6(7):e1000097. Disponível em:

https://doi.org/10.1371/journal.pmed.1000097.

11. Santos CMC, Pimenta CAM, Nobre MRC. The PICO strategy for the research question construction and evidence search. Rev Lat Am Enfermagem [Internet]. 2014 [acesso em: 16 ago. 2018];15(3):508-11. Disponível em: https://doi.org/10.1590/s010411692007000300023.

12. The Joanna Briggs Institute. The JBI Approach - JBI [Internet]. Adelaide: The Joanna Briggs Institute; c2018 [acesso em: 16 ago. 2018]. Disponível em: http://joannabriggs.org/jbi-approach.html\#tabbed-nav=Levels-of-Evidence.

13. NANDA. Diagnósticos de Enfermagem da NANDA: definições e classificações: 2015-2017. 10ª ed. Porto Alegre: Artmed; 2010. 14. Race TK, Collier PE. The Hidden Risk of Deep Vein Thrombosis-The Need for Risk Factor Assessment: Case Reviews. Crit Care Nurs Q [Internet]. 2007 [acesso em: 16 ago. 2018];30(3):245-54. Disponível em:

https://doi.org/10.1097/01.CNQ.0000278925.67562.f4. 
15. Castilho DG, Beccaria LM, Pereira RAM, Contrin LM. Fatores de risco adquiridos e profilaxia da trombose venosa profunda em Unidade de Terapia Intensiva. Arq ciênc saúde [Internet]. 2010 [acesso em: 16 ago. 2018];17(4):169-73. Disponível em:

http://repositorio-racs.famerp.br/racs ol/vol-17-4/IDR\%201.pdf.

16. Ayhan H, Iyigun E, Ince S, Can MF, Hatipoglu S, Saglam M. A randomised clinical trial comparing the patient comfort and efficacy of three different graduated compression stockings in the prevention of postoperative deep vein thrombosis. J Clin Nurs [Internet]. 2015 [acesso em: 16 ago. 2018];24(15-16):2247-57. Disponível em: https://doi.org/10.1111/jocn.12866.

17. Hou LL, Yao LW, Niu QN, Xu L, Yu QH, Sun WQ, et al. Preventive Effect of Electrical Acupoint Stimulation on Lower-Limb Thrombosis: Prospective Study of Elderly Patients After Malignant Gastrointestinal Tumor Surgery. Cancer Nurs [Internet]. 2013 [acesso em: 16 ago. 2018];36(2):139-144. Disponível em: https://doi.org/10.1097/NCC.0b013e3182483415.

18. Ayatollahzade-Isfahani F, Pashang M, Omran AS, Saadat S, Shirani S, Fathollahi MS. Comparing the impact of supine and leg elevation positions during coronary artery bypass graft on deep vein thrombosis occurrence: a randomized clinical trial study. J Vasc Nurs [Internet]. 2013 [acesso em: 16 ago. 2018];31(2):64-7. Disponível em: https://doi.org/10.1016/j.jvn.2012.08.003. 19. Palamone J, Brunovsky S, Groth M, Morris L, Kwasny M. "Tap and twist": preventing deep vein thrombosis in neuroscience patients through foot and ankle range-of-motion exercises. J Neurosci Nurs [Internet]. 2011 [acesso em: 16 ago. 2018];43(6):30814. Disponível em: https://doi.org/10.1097/JNN.0b013e318234e9f2.

20. Le Sage S, McGee M, Emed JD. Knowledge of Venous Thromboembolism (VTE) prevention among hospitalized patients. J Vasc Nurs [Internet]. 2008 [acesso em: 16 ago. 2018];26(4):109-17. Disponível em: https://doi.org/10.1016/j.jvn.2008.09.005.

21. Franks PJ, Barker J, Collier M, Gethin G, Haesler E, Jawien A, et al. Management of Patients With Venous Leg Ulcers: Challenges and Current Best Practice. J Wound Care [Internet]. 2016 [acesso em: 16 ago. 2018];25 Suppl 6:S1-S67. Disponível em: https://doi.org/10.12968/jowc.2016.25.Sup6.S1.

22. Azevedo C, Mata LRF, Faleiro JC, Ferreira MA, Oliveira SP, Carvalho EC. Classificação de intervenções de enfermagem para planejamento de alta médica a pacientes com estomias intestinais. Revista de Enfermagem UFPE Online [Internet]. 2016 [acesso em: 16 ago. 2018];10(2): 531-8. Disponível em: https://periodicos.ufpe.br/revistas/revistaenfermagem/article/view/10986. 23. Caprini JA. Identification of Patient Venous Thromboembolism Risk Across the Continuum of Care. Clin Appl Thromb Hemost [Internet]. 2011 [acesso em: 16 ago. 2018];17(6):590-9. Disponível em: https://doi.org/10.1177/1076029611404217.

24. Sociedade Brasileira de Angiologia e Cirurgia Vascular. Normas de orientação clínica para prevenção, diagnós- tico e tratamento da trombose venosa profunda. J Vasc Br [Internet]. 2005 [acesso em: 16 ago. 2018]; 4(3 Supl. 3):S205-20. Disponível em:

http://jvascbras.com.br/pdf/vol4 n4 supl3.pdf.

25. Yates M, Reddy M, Machumpurath B, Phelps G, Hampson SA. Modification of the National Inpatient Medication Chart improves venous thromboembolism prophylaxis rates in high-risk medical patients. Intern Med J [Internet]. 2014 [acesso em: 16 ago.

2018];44(2):190-4. Disponível em: https://doi.org/10.1111/imj.12346.

26. Duff J, Walker K, Omari A. Translating Venous Thromboembolism (VTE) Prevention Evidence into Practice: A Multidisciplinary Evidence Implementation Project. Worldviews Evid Based Nurs [Internet]. 2011 [acesso em: 16 ago. 2018];8(1):30-9. Disponível em: https://doi.org/10.1111/j.1741-6787.2010.00209.x.

27. Cassidy MR, Rosenkranz P, McAneny D. Reducing Postoperative Venous Thromboembolism Complications with a Standardized Risk-Stratified Prophylaxis Protocol and Mobilization Program. J Am Coll Surg [Internet]. 2014 [acesso em: 16 ago.

2018];218(6):1095-104. Disponível em: https://doi.org/10.1016/j.jamcollsurg.2013.12.061.

28. Lim CS, Davies AH. Graduated compression stockings. CMAJ [Internet]. 2014 [acesso em: 16 ago. 2018];186(10):E391-8.

Disponível em: https://doi.org/10.1503/cmaj.131281.

29. Autar R. A review of the evidence for the efficacy of Anti-Embolism Stockings (AES) in Venous Thromboembolism (VTE) prevention. J Orthop Nurs [Internet]. 2009 [acesso em: 16 ago. 2018];13(1):41-9. Disponível em:

https://doi.org/10.1016/i.joon.2009.01.003.

30. Martin T, Oliver C. Prevention of deep vein thrombosis and pulmonary embolus. Anaesth Intensive Care Med [Internet]. 2009 [acesso em: 16 ago. 2018];10(12):580-2. Disponível em: https://doi.org/10.1016/j.mpaic.2009.09.005.

31. National Institute for Health and Clinical Excellence. Venous thromboembolism: reducing the risk of venous thromboembolism (deep vein thrombosis and pulmonary embolism) in patients admitted to hospital [Internet]. London (UK): Royal College of Physicians; 2010 [acesso em: 16 ago. 2018]. Disponível em: https://www.ncbi.nlm.nih.gov/pubmed/23346611. 32. Hajibandeh S, Antoniou GA, Scurr JR, Torella F. Neuromuscular electrical stimulation for the prevention of venous thromboembolism. Cochrane Database Syst Rev [Internet]. 2015 [acesso em: 16 ago. 2018];(6). Disponível em: https://doi.org/10.1002/14651858.CD011764.

33. Rigon AG, Neves ET. Educação em saúde e a atuação de enfermagem no contexto de unidades de internação hospitalar: o que tem sido ou há para ser dito? Texto contexto - enferm [Internet]. 2011 [acesso em: 16 ago. 2018];20(4):812-7. Disponível em: https://doi.org/10.1590/S0104-07072011000400022.

34. Marini BL, Funk K, Kraft MD, Fong JM, Naanos R, Stout SM, et al. The effects of an informational video on patient knowledge, satisfaction and compliance with venous thromboembolism prophylaxis: a pilot study. Patient Educ Couns [Internet]. 2014 [acesso em: 16 ago. 2018];96(2):264-7. Disponível em: https://doi.org/http://dx.doi.org/10.1590/S0104-07072011000400022. 
Barp M, Carneiro VSM, Amaral KVA, Pagotto V, Malaquias SG.

35. Santos MVR, Oliveira DC, Arraes LB, Oliveira DAGC, Medeiros L, Novaes MA. Adesão ao tratamento anti-hipertensivo: conceitos, aferição e estratégias inovadoras de abordagem. Revista da Sociedade Brasileira de Clínica Médica [Internet]. 2013 [acesso em: 16 ago. 2018];11(1):55-61. Disponível em: http://files.bvs.br/upload/S/1679-1010/2013/v11n1/a3390.pdf. 